\title{
A Novel Design Approach for NB-IoT Networks Using Hybrid Teaching-Learning Optimization
}

\author{
Sotirios K. Goudos ${ }^{1}$, Margot Deruyck ${ }^{2}$, David Plets ${ }^{2}$, Luc Martens ${ }^{2}$, Wout Joseph ${ }^{2}$ \\ ${ }^{1}$ Department of Physics, Aristotle University of Thessaloniki, Thessaloniki 54124, Greece, Email: sgoudo@physics.auth.gr \\ ${ }^{2}$ Department of Information Technology, Ghent University, Ghent, Belgium \\ Email: \{margot.deruyck, david.plets,luc.martens, wout.joseph\}@intec.ugent.be
}

\begin{abstract}
In this paper, we present and address the problem of designing green LTE networks with Internet of Things (IoT) nodes. We consider the new NarrowBand-IoT (NB-IoT) wireless technology that will emerge in current and future access networks. The main objective is to reduce power consumption by responding to the instantaneous bit rate demand by the user and the IoT node. In this context, we apply emerging evolutionary algorithms to the above problem. More specifically, we apply the Teaching-Learning-Optimization (TLBO), the Jaya algorithm, and a hybrid algorithm. This hybrid algorithm named TLBOJaya uses concepts from both algorithms in an effective way. We compare and discuss the preliminary results of these algorithms.
\end{abstract}

Index Terms-NB-IoT, LTE, power consumption, green networks, evolutionary algorithms, teaching- learning optimization.

\section{INTRODUCTION}

Recently in June 2016, 3GPP released the first version of the NarrowBand-IoT (NB-IoT)[1], [2]. NB-IoT is an emerging new wireless access technology, which will exist together with the other existing cellular networks like GSM, UMTS and LTE. The main concept from 3GPP standards is the integration of NB-IoT to current LTE networks. NB-IoT devices will be low cost, that will allow massive deployments and reduced data rates [3]. The carrier bandwidth will be $180 \mathrm{KHz}$ in case of co-existence with current LTE networks. Ericsson predicts that the number of IoT connected devices will reach 1.5 billions by 2022 [4]. NB-IoT massive deployments will create the problem of optimal coverage and power consumption from the access network point of view.

In [5] a capacity-based heuristic is presented for energyefficient network. Moreover, the application of evolutionary algorithms (EAs) to LTE network optimization is also addressed in previous works [6], [7], [8]. In this paper, we consider LTE networks with massive deployment of NB-IoT devices. We optimize the LTE network towards both optimal coverage and power consumption. We consider an optimization approach, which combines an EA together with concepts taken from the capacity tool. We will use three state-of-the-art algorithms that have been recently applied to a wireless sensor network (WSN) optimization problem [9]. These are the TeachingLearning-Optimization (TLBO) [10], the Jaya algorithm, [11] and the recently proposed hybrid TLBO-Jaya [9]. The main feature of all these algorithms is the fact that they do not

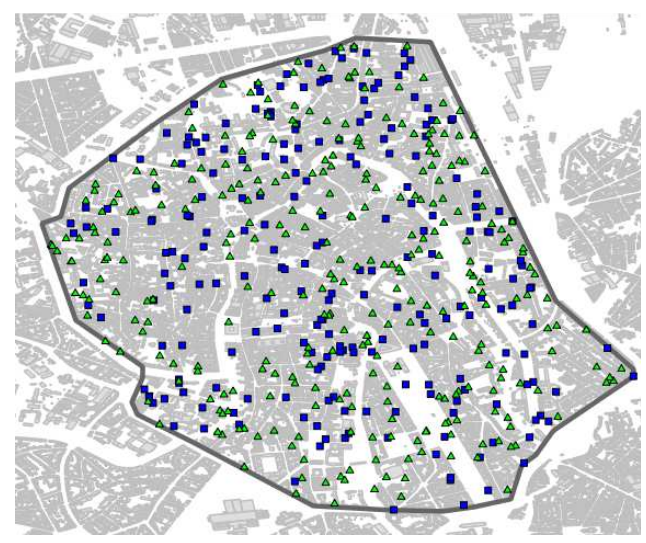

(a)

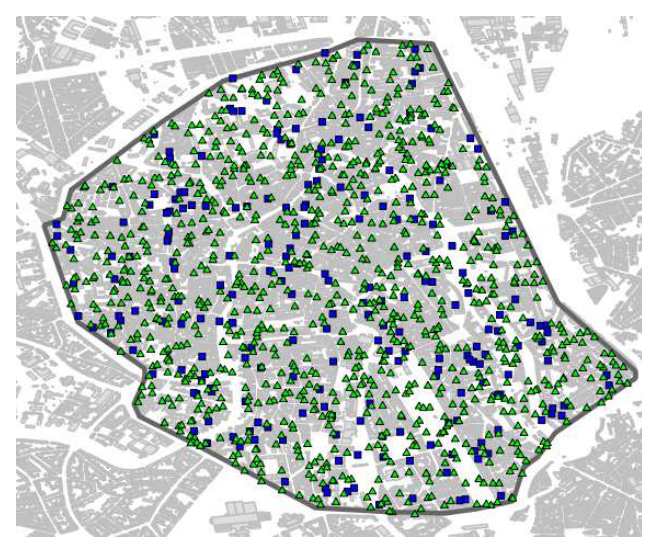

(b)

Fig. 1. Distribution of BS input power of the best obtained solutions. For 224 Voice/Data users+ a) 300 IoT Nodes at $10 \mathrm{kbps}$ b) 300 IoT Nodes at $25 \mathrm{kbps}$

need any control parameter setting other than population size and iteration number. It must be pointed out that in [9] the algorithms were applied in real-valued optimization problem, while in our case they are applied in a discrete-valued problem.

We optimize a LTE network with different NB-IoT node number and data rates. The application area of all the algorithms is the center of Ghent, Belgium. Based on the obtained results, a comparison is made between both the energy and the network performance of all algorithms. To the best of the authors knowledge, this is the first time that a NB-IoT network 
optimization takes place. Moreover, it is the first time that the above presented algorithms are applied to a propagation problem.

The rest of this paper is organized as follows. The problem description is given in Section II.Section III describes briefly the algorithm used. Section IV, presents the numerical results. Finally, we give the conclusion in Section V.

TABLE I

LINK BUDGET PARAMETERS FOR THE NETWORK

\begin{tabular}{|l|l|}
\hline \multicolumn{1}{|c|}{ Parameter } & \multicolumn{1}{|c|}{ Macrocell BS } \\
\hline Frequency & $2.1 \mathrm{GHz}$ \\
\hline $\begin{array}{l}\text { Maximum input } \\
\text { power base sta- } \\
\text { tion antenna }\end{array}$ & $46 \mathrm{dBm}$ \\
\hline $\begin{array}{l}\text { Antenna gain of } \\
\text { base station }\end{array}$ & $18 \mathrm{dBi}$ \\
\hline $\begin{array}{l}\text { Antenna gain of } \\
\text { receiver }\end{array}$ & $0 \mathrm{dBi}$ \\
\hline $\begin{array}{l}\text { Feeder loss base } \\
\text { station }\end{array}$ & $2 \mathrm{~dB}$ \\
\hline $\begin{array}{l}\text { Feeder loss re- } \\
\text { ceiver }\end{array}$ & $0 \mathrm{~dB}$ \\
\hline Fade margin & $10 \mathrm{~dB}$ \\
\hline $\begin{array}{l}\text { Interference mar- } \\
\text { gin }\end{array}$ & $2 \mathrm{~dB}$ \\
\hline $\begin{array}{l}\text { Noise figure of } \\
\text { receiver }\end{array}$ & $8 \mathrm{~dB}$ \\
\hline $\begin{array}{l}\text { Implementation } \\
\text { loss of receiver }\end{array}$ & $0 \mathrm{~dB}$ \\
\hline MIMO None \\
\hline $\begin{array}{l}\text { Height } \\
\text { station }\end{array}$ & 10.5 for Voice/Data \\
\hline $\begin{array}{l}\text { Building penetra- } \\
\text { tion loss }\end{array}$ & 0 \\
outdoor coverage \\
considered)
\end{tabular}

\section{Problem Formulation}

We address network planning optimization for the LTE NBIoT Network, given 75 possible base stations (BSs) locations in the city center of Ghent, Belgium. The total area to cover is about $6.85 \mathrm{~km}^{2}$. In this paper, we assume a carrier frequency of $2.1 \mathrm{GHz}$ for all the simulations, which is one of the proposed frequency bands for NB-IoT operation [1]. NB-IoT can be deployed in three different operation modes. These are stand-alone as a dedicated carrier, in-band within the occupied bandwidth of a wideband LTE carrier, and within the guardband of an existing LTE carrier. In this case, we consider the in-band operation and thus assume a bandwidth of
$180 \mathrm{KHz}$. The result of the reduced bandwidth is the low data rates supported by NB-IoT devices. In this paper, we consider data rates of $10 \mathrm{kbps}$ and $25 \mathrm{kbps}$ (maximum data rate). Table I holds the link budget parameters for the LTE NB-IoT network. Moreover, we apply the Walfisch-Ikegami propagation model for all path loss calculations in the LTE network.

\section{A. Optimization procedure}

The input to the optimization algorithm is the list of all BSs in the city of Ghent, and the list users of different types (voice/data/IoT nodes). The list of users is generated randomly from a uniform distribution and they are spread in all parts of the city. The input file contains the user location information along with the required bit rate. We consider 224 voice/data users that require $64 \mathrm{kbps} / 1 \mathrm{Mbps}$, respectively. Additionally, the user file contains 300 or 1000 IoT nodes spread randomly. Figs 1a-1b show the user distribution in the city of Ghent for both cases. In these the green triangles represent the IoT nodes, while the blue squares are the voice/data users. The solution vector $\bar{y}$ is an integer vector that contains both the active BSs and the operating power in $\mathrm{dBm}$. This vector is produced by any of the selected EAs. In this paper, we use the TLBO, Jaya, and the TLBO-Jaya algorithms. This solution vector is the input to another algorithm that calculates an objective function based on the number of covered users and the power consumption of the current solution. We formulate this objective function as

$$
F(\bar{y})=\frac{P(\bar{y})}{P_{\max }}+\Xi \cdot\left(1-\frac{M_{\text {cov }}}{M_{\text {tot }_{u} \text { sers }}}\right)
$$

where $M_{c o v}$ is the number of users covered with a given solution $\bar{y}, M_{\text {tot }_{u} \text { sers }}$ is the total number of users, $P(\bar{y})$ is the total power consumted with this solution, $P_{\max }$ is the maximum power consumption when all BS are active and operate at full power, and $\Xi$ is a very large number. The algorithm works in the following way; For a given solution it calculates the number of users covered by this solution, then it computes the objective function value.

\section{AlgORITHM DESCRIPTION}

In order to select a suitable EA to optimize the network we have chosen algorithms with low complexity. Additionally, a desirable algorithm characteristic is the absence of control parameters, so that no additional fine tuning is required. Therefore, we have selected three parameter-free algorithms. These are the Teaching-Learning-Optimization (TLBO) [10], the Jaya algorithms, [11] and the recently proposed hybrid TLBOJaya [9]. TLBO uses concepts from learning and teaching in a class and it uses two distinct phases. These are called the "teacher phase" (models teaching from a best student), and the "learner phase" (models exchanging information and learning between students). The Jaya algorithm is very simple and uses a simple update equation. This is based on the concept that we need to move towards the best solution and away from the worst solution. The TLBO-Jaya algorithm, which 


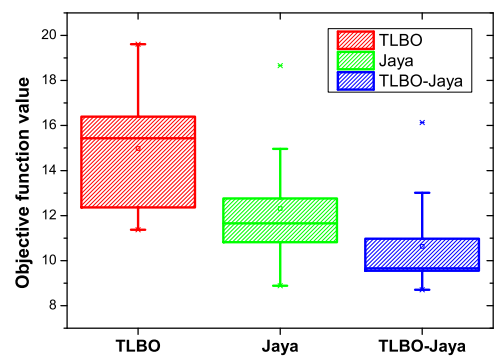

(a)

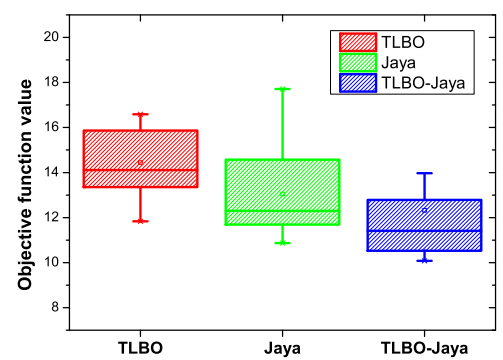

(b)

Fig. 2. Box plots of the algorithms results.For 224 Voice/Data users and a) 300 IoT Nodes at $10 \mathrm{kbps}$ b) 300 IoT Nodes at $25 \mathrm{kbps}$

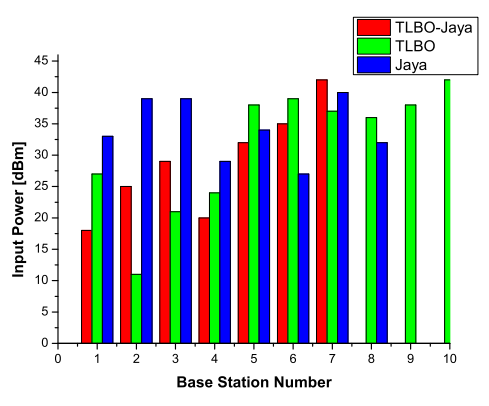

(a)

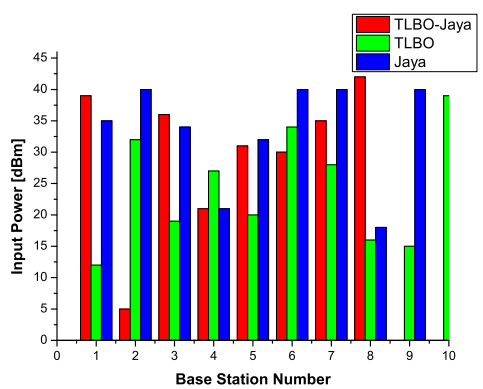

(b)

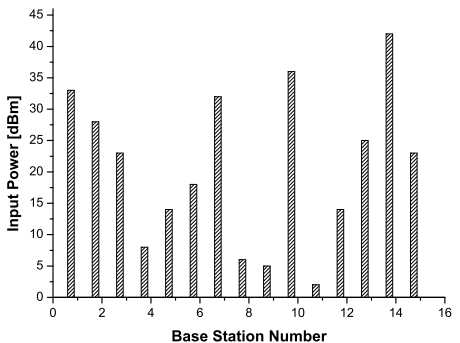

(c)

Fig. 3. Distribution of BS input power of the best obtained solutions. For 224 Voice/Data users and a) 300 IoT Nodes at $10 \mathrm{kbps}$ b) 300 IoT Nodes at $25 \mathrm{kbps}$ c) 1000 IoT Nodes at $25 \mathrm{kbps}$.

was introduced in [9], can be described with the following steps.

Step 1. We select the population size Pop, and the maximum number of objective function evaluations $M A X F E S$.

Step 2. A random population of Pop solution vectors (BSs power distribution) is generated by a uniform distribution.

Step 3. As in original TLBO first its the teacher phase. Here, we calculate first the population mean M. Then TLBOJaya calculates a teaching factor,$T_{f}$, in a different way than the original TLBO. This is done by using the expression

$$
T_{f}=\text { round }\left(1+\frac{F_{k}-F_{\text {best }}}{F_{\text {worst }}-F_{\text {best }}}\right)
$$

where $F_{k}$ is the objective function value of the $k-t h$ solution and $F_{\text {best }}, F_{\text {worst }}$ are the minimum and maximum function values obtained so far among all solutions, respectively. Then a new solution vector is generated by

$$
\mathbf{y}_{\mathbf{k}}^{\text {new }}=\mathbf{y}_{\mathbf{k}}^{\text {old }}+\operatorname{rand}_{k}\left(\mathbf{y}_{\text {best }}-T_{f} \mathbf{M}\right)
$$

where $\operatorname{rand}_{k}$ is a uniformly distributed random number within the range $[0,1]$. A greedy selection scheme is then applied to the new vector, where the new vector replaces the old in the next iteration only if its objective function is better than the old.

Step 4. The learner phase is next. In this step the algorithm selects two random learners $k$ and $m$ from the population.
These exchange information with each other and generate a new vector $\mathbf{y}_{\mathbf{y}}$ that is defined as

$$
\begin{aligned}
& \mathbf{y}_{\mathbf{k}}^{\text {new }}=\mathbf{y}_{\mathbf{k}}^{\text {old }}+\operatorname{rand}_{k}\left(\mathbf{y}_{\mathbf{k}}-\mathbf{y}_{\mathbf{m}}\right), \text { if } F\left(\mathbf{y}_{\mathbf{k}}\right)<F\left(\mathbf{y}_{\mathbf{m}}\right) \\
& \mathbf{y}_{\mathbf{k}}^{\text {new }}=\mathbf{y}_{\mathbf{k}}^{\text {old }}+\operatorname{rand}_{k}\left(\mathbf{y}_{\mathbf{m}}-\mathbf{y}_{\mathbf{m}}\right), \text { otherwise }
\end{aligned}
$$

where $r_{k}$ is a random number within the range [0,1].

Step 5. The Jaya phase where a new vector is generated using the concepts from the Jaya algorithm, which is defined as

$\mathbf{y}_{\mathbf{i}}^{\text {new }}=\mathbf{y}_{\mathbf{k}}^{\text {old }}+\operatorname{rand}_{1}\left(\mathbf{y}_{\text {best }}-\mathbf{y}_{\mathbf{k}}^{\text {old }}\right)-\operatorname{rand}_{2}\left(\mathbf{y}_{\text {worst }}-\mathbf{y}_{\mathbf{k}}^{\text {old }}\right)$

where $\operatorname{rand}_{1}$ and $\operatorname{rand}_{2}$ are uniformly distributed random numbers within the range $[0,1]$. Again greedy selection scheme is applied to the new vector.

Step 6. The algorithm stops if the number of objectivefunction evaluations becomes larger than $M A X F E S$.

\section{NuMERICAL RESULTS}

We compare different LTE NB-IoT network configurations with 75 possible BSs. The BS can be either active (binary one) or not (binary zero). When a BS operates then the range of the input power of the BS antenna is between 0 to 46 $\mathrm{dBm}$, with a step of $1 \mathrm{dBm}$. In all presented cases, we assume macrocell BSs. We consider three different user distribution cases. In all cases we consider 224 voice/data users with rates 
of $64 \mathrm{kbps} / 1 \mathrm{Mbps}$ respectively. Additionally, we consider a large number of NB-IoT nodes spread in the city center. The first case consists of 300 IoT nodes with a rate of $10 \mathrm{kbps}$. It must be noted that the number 300 is in accordance with a typical number of nodes for smart city applications as it is reported in [12]. The second network case is the same as in previous with the exception of the downlink speed. This is set to $25 \mathrm{kbps}$ for all nodes, which is the maximum speed according to 3GPP standards. The final case is a new user distribution consisting of 1000 IoT nodes. For the first two cases, we compare results with the three algorithms, namely TLBO, Jaya, and TLBO-Jaya. In the last case, we optimize the network using the TLBO-Jaya algorithm. The total number of unknowns is $2 \times 75$ for all cases. We run the TLBO, Jaya and TLBO-Jaya algorithms 10 times. The best results are obtained. We select the population size to be 10 and the maximum number of generations is 30 for all algorithms. Therefore, the total number of objective function evaluations is 300 .

TABLE II

ALGORITHMS COMPARATIVE RESULTS FOR ALL CASES. THE SMALLER VALUES ARE IN BOLD FONT.

\begin{tabular}{|r|c|c|c|c|}
\hline \multicolumn{5}{|c|}{300 nodes 10Kbps } \\
\hline Algorithm & Best & Worst & Mean & Std. Dev. \\
\hline TLBO & 11.37 & 19.61 & 14.98 & 2.46 \\
\hline Jaya & 8.88 & 18.66 & 12.32 & 2.64 \\
\hline TLBO-Jaya & $\mathbf{8 . 7 0}$ & $\mathbf{1 6 . 1 3}$ & $\mathbf{1 0 . 6 3}$ & $\mathbf{2 . 1 8}$ \\
\hline \multicolumn{5}{|c|}{$\mathbf{3 0 0}$ nodes 25Kbps } \\
\hline Algorithm & Best & Worst & Mean & Std. Dev. \\
\hline TLBO & 11.84 & $\mathbf{1 6 . 5 8}$ & 14.44 & $\mathbf{1 . 5 1}$ \\
\hline Jaya & 10.86 & 17.71 & 13.05 & 2.04 \\
\hline TLBO-Jaya & $\mathbf{1 0 . 0 8}$ & 19.58 & $\mathbf{1 2 . 3 2}$ & 2.66 \\
\hline
\end{tabular}

Table II reports the comparative results for all algorithms in terms of objective function values. We notice that TLBOJaya obtains the best results in both cases. Moreover, Jaya emerged as the second best algorithm. For the second case the TLBO obtained the smaller standard deviation value. Figs. 2a$2 \mathrm{~b}$ illustrate the box plots of all the algorithms runs. In the first case, TLBO-Jaya obtained the narrower distribution of values, while maintained the smaller values. For the second case all algorithms seem to have produced similar distributions, however again TLBO-Jaya obtained smaller values.

The best obtained values regarding the network design are reported in Table III. We notice that for the first case TLBO-Jaya obtained a solution with $7 \mathrm{BS}$, while the other algorithms obtained best solutions with more BSs. There is a large difference in the power consumption also among the algorithms. The capacity column refers to the total capacity in Mbps offered by each network configuration regardless of user coverage. For the second case again TLBO-Jaya achieves a solution with fewer active BSs than the other algorithms. The final case is that of a network configuration with 1000 IoT nodes. We notice that TLBO-Jaya increased the number of BSs from 8 to 15 (about doubled) for covering three times
TABLE III

BEST-OBTAINED RESULTS COMPARISON USING DIFFERENT ALGORITHMS. THE SMALLER VALUES ARE IN BOLD FONT.

\begin{tabular}{|c|c|c|c|c|}
\hline \multicolumn{5}{|c|}{300 IoT nodes $10 \mathrm{Kbps}+224$ Voice-Data Users } \\
\hline Algorithm & BS No. & $\begin{array}{l}\text { Served } \\
\text { Users } \\
(\%)\end{array}$ & $\begin{array}{l}\text { Power } \\
\text { Con- } \\
\text { sump. } \\
(\mathrm{kW})\end{array}$ & $\begin{array}{l}\text { Capacity } \\
\text { (Mbps) }\end{array}$ \\
\hline Jaya & 8 & 100 & 10.42 & 108.80 \\
\hline TLBO & 10 & 100 & 13.14 & 136.00 \\
\hline $\begin{array}{l}\text { TLBO- } \\
\text { Jaya }\end{array}$ & 7 & 100 & 8.95 & 95.20 \\
\hline \multicolumn{5}{|c|}{300 IoT nodes $25 \mathrm{Kbps}+\mathbf{2 2 4}$ Voice-Data Users } \\
\hline Algorithm & BS No. & $\begin{array}{l}\text { Served } \\
\text { Users } \\
(\%)\end{array}$ & $\begin{array}{l}\text { Power } \\
\text { Con- } \\
\text { sump. } \\
\text { (kW) }\end{array}$ & $\begin{array}{l}\text { Capacity } \\
\text { (Mbps) }\end{array}$ \\
\hline Jaya & 9 & 100 & 11.96 & 122.40 \\
\hline TLBO & 10 & 100 & 12.36 & 135.99 \\
\hline $\begin{array}{l}\text { TLBO- } \\
\text { Jaya }\end{array}$ & 8 & 100 & 10.42 & 108.79 \\
\hline \multicolumn{5}{|c|}{1000 IoT nodes $25 \mathrm{Kbps}+224$ Voice-Data Users } \\
\hline $\begin{array}{l}\text { TLBO- } \\
\text { Jaya }\end{array}$ & 15 & 100 & 18.66 & 203.999 \\
\hline
\end{tabular}

more nodes. The distribution of BS input power of the best obtained solution is depicted in Figs. 3a-3c. We notice that the $\mathrm{BS}$ input power varies and is not always set to maximum power.

\section{CONCLUSiON}

NB-IoT technology will soon be part of every current LTE network. In this paper, we have introduced and addressed the problem of designing LTE NB-IoT networks for the best possible coverage and optimal power consumption. Moreover, we have applied a new and simple algorithm to this problem, the TLBO-Jaya algorithm. This algorithm was compared with others in two different network cases. The results indicate that it obtained a better performance than other emerging algorithms. In our future work, we plan to design NB-IoT networks in a 5G Massive MIMO environment.

\section{REFERENCES}

[1] 3GPP, "Tr 45.820 cellular system support for ultra-low complexity and low throughput internet of things (ciot), relase 13," Tech. Rep., 2015.

[2] — , "Ts 36.802, evolved universal terrestrial radio access (e-utra); nb-iot; technical report for bs and ue radio transmission and reception (release 13)," Tech. Rep., 2016.

[3] A. D. Zayas and P. Merino, "The 3gpp nb-iot system architecture for the internet of things," in 2017 IEEE International Conference on Communications Workshops (ICC Workshops), May 2017, pp. 277-282.

[4] Ericsson, "Ericsson Mobility Report June 2017," White paper, 2017. [Online]. Available: https://www.ericsson.com/en/mobility-report

[5] M. Deruyck, W. Joseph, E. Tanghe, and L. Martens, "Reducing the power consumption in lte-advanced wireless access networks by a capacity based deployment tool," Radio Science, vol. 49, no. 9, pp. 777787,2014

[6] M. Deruyck, D. Plets, L. Martens, W. Joseph, and S. Goudos, "Optimizing wireless access networks towards power consumption: Influence of the optimization algorithm," 2016, pp. 261-265. 
[7] S. Goudos, M. Deruyck, D. Plets, L. Martens, and W. Joseph, "Optimization of power consumption in $4 \mathrm{~g}$ lte networks using a novel barebones self-adaptive differential evolution algorithm," Telecommunication Systems, vol. 66, no. 1, pp. 109-120, 2017.

[8] — "Application of opposition-based learning concepts in reducing the power consumption in wireless access networks," 2016.

[9] A. Tsiflikiotis, S. Goudos, and G. Karagiannidis, "Hybrid teachinglearning optimization of wireless sensor networks," Transactions on Emerging Telecommunications Technologies, 2017.

[10] R. V. Rao, V. J. Savsani, and D. P. Vakharia, "Teaching-learningbased optimization: A novel method for constrained mechanical design optimization problems," Computer-Aided Design, vol. 43, no. 3, pp. 303 315, 2011.

[11] R. V. Rao, "Jaya : A simple and new optimization algorithm for solving constrained and unconstrained optimization problems," International Journal of Industrial Engineering Computations, vol. 7, no. 1, pp. 1934, 2016.

[12] A. Zanella, N. Bui, A. Castellani, L. Vangelista, and M. Zorzi, "Internet of things for smart cities," IEEE Internet of Things Journal, vol. 1, no. 1, pp. 22-32, 2014 